\title{
Scaling law for the photon spectral density in the nonlinear Thomson-Compton scattering
}

\author{
D. Seipt* and B. Kämpfer ${ }^{\dagger}$ \\ Helmholtz-Zentrum Dresden-Rossendorf, P.O. Box 5101 19, 01314 Dresden, Germany
}

(Received 2 July 2010; published 12 April 2011)

\begin{abstract}
We derive a new scaling law for the photon spectral density in nonlinear Thomson/Compton scattering, extending the findings of Heinzl, Seipt, and Kämpfer [Phys. Rev. A 81, 022125 (2010)]. This allows one to easily include the effects of general scattering geometries, e.g., side injection, and of a finite-size detector on the photon spectrum. The scaling law is employed to study substructures emerging in the nonlinear Thomson/Compton spectra due to temporally shaped laser pulses scattering off relativistic electrons. We determine optimum scattering geometries for an experimental verification of these substructures.
\end{abstract}

DOI: 10.1103/PhysRevSTAB.14.040704

PACS numbers: 12.20.Ds, 41.60. $-\mathrm{m}$

\section{INTRODUCTION}

The nonlinear Thomson/Compton scattering-the first being a low-energy (low-recoil) limit of the latter [1]—of intense laser photons off relativistic electrons has attracted much attention both theoretically (predicting effects like the emission of harmonic radiation or the red shift of the Compton edge [2-4]), and experimentally (trying to measure the predicted effects $[5,6])$. Most of the theoretical works consider plane-wave laser fields or box-shaped pulses, with a few exceptions $[1,7,8]$. In the latter references, it was shown that the emitted spectrum in the nonlinear regime is significantly modified when considering smooth finite laser pulses, which cause various substructures in the spectrum. This phenomenon may be named ponderomotive broadening. The substructures have not been observed in an experiment yet, presumably due to the demanding requirements posed on the quality of the electron beam concerning, in particular, low emittance and low energy spread.

Here, we extend the analysis of [1] and derive a more general scaling law for the Thomson/Compton backscattered photon spectrum. The scaling law allows an efficient calculation of the photon spectra emitted by relativistic electrons in a laser pulse with finite duration. General geometries with respect to the electron beam direction and the laser beam direction as well as the emission (observation) direction and the electron phase-space distribution are accounted for. (The scaling law in [1] was for strict backscattering in head-on collisions only.) The photon spectrum is easily integrated over a range of emission angles, thus properly simulating an energy sensitive detector of finite size.

\footnotetext{
*d.seipt@hzdr.de

†b.kaempfer@hzdr.de
}

Published by American Physical Society under the terms of the Creative Commons Attribution 3.0 License. Further distribution of this work must maintain attribution to the author(s) and the published article's title, journal citation, and DOI.

\section{THOMSON/COMPTON SCATTERING KINEMATICS}

Let us consider Thomson/Compton scattering of an ultraintense laser off relativistic electrons. A convenient measure for the laser intensity is the dimensionless amplitude $a_{0}=e E / m \omega$ with the rms electric field $E$, charge $e$, electron mass $m$, and laser-photon frequency $\omega$. The energy momentum conservation for this process reads

$$
\ell k+q=k^{\prime}+q^{\prime},
$$

where $k\left(k^{\prime}\right)$ is the incoming (outgoing) photon 4-momentum and $q=p+k\left[\left(a_{0}^{2} m^{2}\right) /(4 k \cdot p)\right]\left(q^{\prime}=p^{\prime}+\right.$ $\left.k\left[\left(a_{0}^{2} m^{2}\right) /\left(4 k \cdot p^{\prime}\right)\right]\right)$ denotes the incoming (outgoing) electron quasimomentum. Using $k^{2}=k^{12}=0, q^{2}=q^{12}$, and $q \cdot k=p \cdot k,(1)$ yields

$$
\ell k \cdot p=k^{\prime} \cdot p+k^{\prime} \cdot k\left(\ell+\frac{a_{0}^{2} m^{2}}{4 k \cdot p}\right)
$$

where the $\ell$ in the brackets may be neglected in the Thomson limit $k \cdot p / m^{2} \ll 1$ (the quantity $\ell$ refers to the harmonic; a dot denotes the scalar product of 4-vectors). With the definitions $k=\omega n, k^{\prime}=\omega^{\prime} n^{\prime}$, and $p=m u$, one obtains from (1) the frequency of scattered photons as

$$
\omega_{\ell}^{\prime}\left(n^{\prime}, u\right)=\frac{\ell \omega n \cdot u}{n^{\prime} \cdot u+n \cdot n^{\prime} \frac{a_{0}^{2}}{4 n \cdot u}}=: \omega \chi_{\ell}\left(n^{\prime}, u\right) .
$$

This defines a mapping function $\chi_{\ell}$ for nonlinear Thomson scattering representing the Doppler upshift of the frequency $\omega$. In the linear regime, $a_{0} \ll 1$, it condenses to $\chi=n \cdot u / n^{\prime} \cdot u$. The kinematics, i.e., the incoming and outgoing momenta, are specified and parametrized by

$$
\begin{gathered}
n=(1, \mathbf{n}), \quad n^{\prime}=\left(1, \mathbf{n}^{\prime}\right), \quad u=(\gamma, \mathbf{u}), \\
\mathbf{n}=(0,0,-1), \\
\mathbf{n}^{\prime}=(\sin \theta \cos \phi, \sin \theta \sin \phi, \cos \theta), \\
\mathbf{u}=\gamma \beta(\sin \alpha \cos \varphi, \sin \alpha \sin \varphi, \cos \alpha),
\end{gathered}
$$


with $\gamma=p^{0} / m, \beta=\sqrt{\gamma^{2}-1} / \gamma$, and $p^{0}$ as energy of the electron. (For a visualization, see Fig. 9 of [1].) Here, the angles $(\alpha, \varphi)$ and $(\theta, \phi)$ measure the direction of incoming electrons and outgoing photons with respect to the $z$ axis, defined by $\mathbf{n}$, respectively. With this choice, the various scalar products in (3) become

$$
\begin{gathered}
n \cdot n^{\prime}=1+\cos \theta, \\
n \cdot u=\gamma(1+\beta \cos \alpha)=: \kappa, \\
n^{\prime} \cdot u=\gamma(1-\beta \cos \bar{\alpha}), \\
\cos \bar{\alpha}=\cos \alpha \cos \theta+\sin \alpha \sin \theta \cos (\varphi-\phi) .
\end{gathered}
$$

For the normalized vector potential of the laser field $a^{\mu}=e A^{\mu} / m$, we choose a temporal gauge $a^{\mu}=(0, \mathbf{a})$ with $\mathbf{a}=a_{0} \varepsilon e^{-i \omega n \cdot x} g_{\sigma}(k \cdot x)$, where $\sigma$ is a dimensionless pulse length parameter, and $\varepsilon$ denotes the normalized real or complex polarization vector

$$
\varepsilon \in \begin{cases}\left\{\mathbf{e}_{x}, \mathbf{e}_{y}\right\} & \text { for linear polarization, } \\ \left\{\frac{\mathbf{e}_{x}+i \mathbf{e}_{y}}{\sqrt{2}} \frac{\mathbf{e}_{x}-i \mathbf{e}_{y}}{\sqrt{2}}\right\} & \text { for circular polarization. }\end{cases}
$$

In our numerical simulations we use a solitonic pulse $g_{\sigma}(k \cdot x)=\cosh ^{-1}(k \cdot x / \sigma)$ through this paper.

\section{PHOTON SPECTRAL DENSITY}

Having defined the kinematics, the spectral density of emitted photons in nonlinear Thomson scattering, i.e., the emission of photons by relativistic electrons (for details of the relation of Thomson and Compton scattering cf. [1]), may be written as [9]

$$
\rho\left(\omega^{\prime} ; n^{\prime}, u\right) \equiv \frac{d^{2} N_{\gamma}}{d \omega^{\prime} d \Omega}=\frac{\omega^{\prime}}{16 \pi^{3}}\left|\mathbf{n}^{\prime} \times \mathbf{j}\left(k^{\prime}\right)\right|^{2}
$$

with the Fourier transform of the electron current

$$
\mathbf{j}\left(k^{\prime} ; u\right)=e \int_{-\infty}^{\infty} d \tau \mathbf{U}(\tau ; u) e^{i k^{\prime} \cdot X(\tau ; u)}
$$

and the electron trajectory $X(\tau)$ and velocity $U(\tau)$ with $u=U\left(\tau_{0}\right)$ as the solution of the relativistic Lorentz force equation $d U^{\mu} / d \tau=f^{\mu \nu} U_{\nu}$, where $f^{\mu \nu}=\partial^{\mu} a^{\nu}-\partial^{\nu} a^{\mu}$. When the fields only depend on one variable (in this case the phase $n \cdot x$ ), the latter one can be calculated completely analytically [10] due to the existence of an additional constant of motion, namely, the light cone variable $n \cdot U(\tau)=n \cdot u=\kappa$ yielding a relation between the plane-wave phase and the electron's proper time $d \tau=$ $d(n \cdot X) / \kappa$. The electron orbits in index notation are given by

$$
\begin{gathered}
U^{\mu}(\tau)=u^{\mu}-a^{\mu}+n^{\mu}\left(\frac{a \cdot u}{n \cdot u}-\frac{a \cdot a}{2 n \cdot u}\right), \\
X^{\mu}(\tau)=\int_{\tau_{0}}^{\tau} d \hat{\tau} U^{\mu}(\hat{\tau}) .
\end{gathered}
$$

For sufficiently weak fields, i.e. $a_{0} \ll 1$, the electron current $\mathbf{j}$ may be linearized in $a_{0}$ by expanding $\exp \left\{i k^{\prime} \cdot X(\tau)\right\} \simeq 1+i k^{\prime} \cdot X(\tau)$ Furthermore, for not too short pulses, e.g. $\sigma \gtrsim 5$, the slowly varying envelope approximation can be applied to the proper-time integral (16) yielding for the electron current

$$
\mathbf{j}\left(\omega^{\prime} ; n^{\prime}, u\right)=-\frac{e a_{0}}{\kappa} \tilde{g}\left(\omega-\frac{\omega^{\prime}}{\chi}\right) \dot{\mathrm{j}}_{0},
$$

with $\tilde{g}$ being the Fourier transform of $g_{\sigma}$. The vector part of the current reads

$$
\begin{gathered}
\dot{j}_{0}=\varepsilon+\mathcal{A} \mathbf{n}+\mathcal{B} \mathbf{u}, \\
\mathcal{A}=(\varepsilon \cdot \mathbf{u}) / \kappa, \\
\mathcal{B}=\frac{\omega^{\prime}}{\omega \kappa}\left(\varepsilon \cdot \mathbf{n}^{\prime}-n \cdot n^{\prime} \mathcal{A}\right),
\end{gathered}
$$

which allows one to cast (13) in the form

$$
\rho\left(\omega^{\prime} ; n^{\prime}, u\right)=\frac{e^{2} a_{0}^{2}}{16 \pi^{3}} \frac{\omega^{\prime}}{\kappa^{2}}\left|\tilde{g}\left(\omega-\frac{\omega^{\prime}}{\chi}\right)\right|^{2}\left|\mathbf{n}^{\prime} \times \dot{j}_{0}\right|^{2} .
$$

Some prominent limits of $\left|\mathbf{n}^{\prime} \times \dot{j}_{0}\right|$ include (i) the backscattering geometry with $\mathbf{n}^{\prime} \cdot \mathbf{n}=-1, \mathbf{n}^{\prime} \cdot \varepsilon^{(*)}=0$ discussed in [1], and (ii) the case where the photon is scattered into the direction of the incoming electron, i.e. $\mathbf{n}^{\prime} \| \mathbf{u}$. The latter case yields the particularly simple result,

$$
\left|\mathbf{n}^{\prime} \times \dot{j}_{0}\right|^{2}=1+\left|\varepsilon \cdot \mathbf{n}^{\prime}\right|^{2}\left\{\lambda^{2}-\left(1+\lambda \mathbf{n} \cdot \mathbf{n}^{\prime}\right)^{2}\right\},
$$

with $\lambda=\gamma \beta / \kappa$ reflecting the high symmetry. This special scattering geometry is considered in Sec. V.

\section{SCALING LAW}

The basic idea behind the scaling law was motivated in [1], where the restrictions of head-on collisions and strict backscattering have been imposed. Both restrictions are relaxed in the present work to deal with arbitrarily inclined electron and laser beams as well as scattering angles.

According to (13) the spectral density $\rho\left(\omega^{\prime} ; n^{\prime}, u\right)$ depends on the initial conditions through the orbit $X(\tau ; u)$ and its velocity $U(\tau ; u)$. The posed problem is how the spectral density changes when changing both the initial conditions and the observation direction from one set of values [e.g. $u_{0}=u_{0}\left(\gamma_{0}, \alpha_{0}, \varphi_{0}\right), n_{0}^{\prime}=n_{0}^{\prime}\left(\theta_{0}, \phi_{0}\right)$ ] to another one [e.g. $\left.u(\gamma, \alpha, \varphi), n^{\prime}(\theta, \phi)\right]$. To formalize this problem we consider such a change as being due to a Lorentz transformation $u_{0} \rightarrow u=\Lambda u_{0}$, which actually depends on the six parameters $\alpha_{0}, \varphi_{0}, \gamma_{0}, \alpha, \varphi, \gamma$ (an explicit expression for $\Lambda$, constructed from pure boosts $\Lambda_{B}$ and pure rotations $\Lambda_{R}$, can be found in [1]), and similarly $n^{\prime}=\Xi n_{0}^{\prime}$ with $\Xi=\Lambda_{R}(\theta, \phi) \Lambda_{R}^{-1}\left(\theta_{0}, \phi_{0}\right)$ being a pure $3 \mathrm{D}$ rotation. 
Armed with this, the scaling relation is found to be

$$
\rho\left(\omega^{\prime} ; n^{\prime}, u\right)=\mathcal{M}\left(u_{0}, u ; n_{0}^{\prime}, n^{\prime}\right) \rho\left(\omega^{\prime} / h ; n_{0}^{\prime}, u_{0}\right),
$$

with the transition function

$$
\mathcal{M}=h \frac{\left(n \cdot u_{0}\right)^{2}}{(n \cdot u)^{2}} \frac{\left|\mathbf{n}^{\prime} \times \dot{j}_{0}\left(h \omega^{\prime} ; u, n^{\prime}\right)\right|^{2}}{\left|\mathbf{n}^{\prime} \times \dot{j}_{0}\left(\omega^{\prime} ; u_{0}, n_{0}^{\prime}\right)\right|^{2}}
$$

and $h=\chi\left(u, n^{\prime}\right) / \chi\left(u_{0}, n_{0}^{\prime}\right)$. Equations (23) and (24) together with the definition of $\dot{j}_{0}$ represent a general formulation of the scaling law for the spectral distribution of photons emitted by an electron in a plane electromagnetic wave, i.e., for Thomson/Compton scattering. The group property of Lorentz transformations is carried over to the scaling transformations. Consider, for example, two subsequent transformations $u_{0} \rightarrow u_{1}=\Lambda_{1} u_{0} \rightarrow u_{2}=$ $\Lambda_{2} u_{1}$, then

$$
\begin{gathered}
h\left(u_{0}, u_{2}\right)=h\left(u_{0}, u_{1}\right) h\left(u_{1}, u_{2}\right), \\
\mathcal{M}\left(u_{0}, u_{2}\right)=\mathcal{M}\left(u_{0}, u_{1}\right) \mathcal{M}\left(u_{1}, u_{2}\right) .
\end{gathered}
$$

The same relations also hold when including transformations of $n^{\prime}$.

In the form given in (23), the scaling law is valid only for $a_{0} \ll 1$. To include the nonlinear case, $a_{0} \gtrless 1$, we finally substitute the nonlinear Doppler factor $\chi_{1}$ [cf. (3)] for the linear Doppler factor $\chi$ in the definition of $h$. In the nonlinear regime, the scaling law perfectly accounts for changes in the electron's initial energy. However, changes of the incidence angles, in particular $\alpha$, are rendered accurate only for $\alpha \ll 1$. Nevertheless, for the present purposes, the scaling law is sufficiently accurate to account for the typical angular divergence of focused electron beams. To check the accuracy of the scaling law, a comparison of a direct numerical integration of (13) and the application of the scaling law (23) is shown in Fig. 1. In the latter case, Eq. (13) has been solved numerically only for one set of parameters (black solid curve) for a given value of $a_{0}$; all black dashed curves have been obtained by employing the scaling law (23). For the employed parameters (see caption of Fig. 1), the black dashed curves are very well on top of the solid colored curves representing the direct numerical evaluations of (13) for each parameter set.

It is important to emphasize that the laser focus waist $w_{0}$ must be substantially larger than the electron-beam radius $r_{b}$, so that a plane-wave description of the laser field may be applied in the relevant interaction region.

\section{ELECTRON PHASE-SPACE DISTRIBUTION}

In general, an electron bunch consists of $N_{e}$ electrons whose individual spectra $\rho\left(\omega^{\prime} ; n^{\prime}, u^{(i)}\right)$, emitted by the $i$ th electron into the direction $n^{\prime}$, have to be added incoherently (as long as the electron density is not exceedingly high) to obtain the total spectral density, which may be dubbed warm spectral density
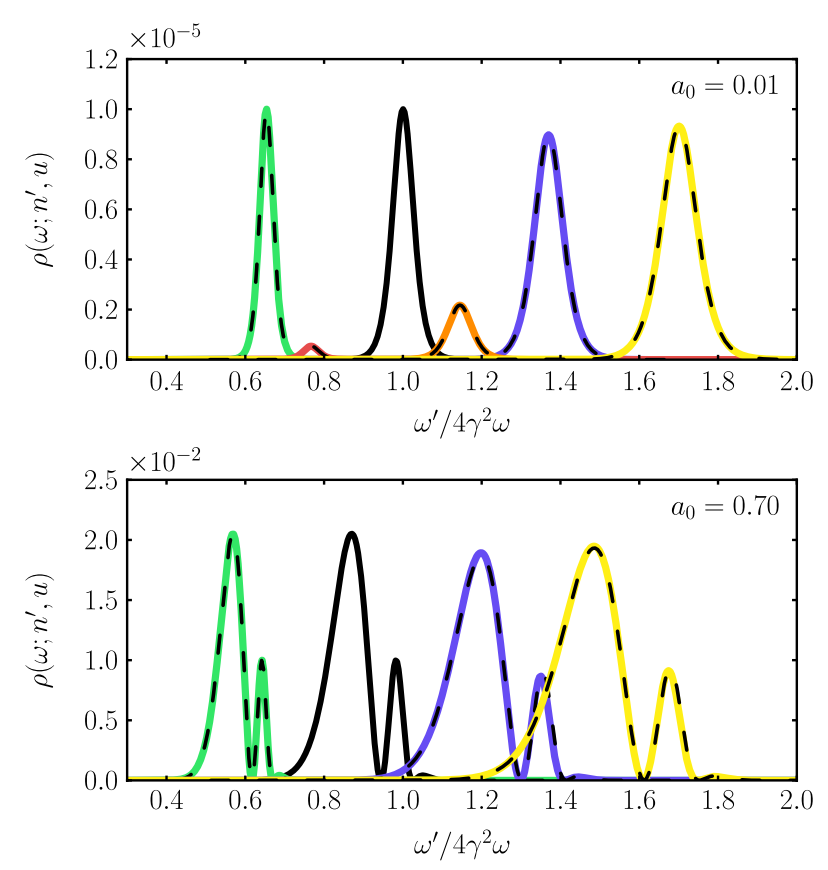

FIG. 1. Photon spectral density $\rho\left(\omega^{\prime}, n^{\prime}, u\right)$ for a single electron by numerical evaluation of Eq. (13) (colored solid curves) and by application of the scaling law (black dashed curves). The upper panel shows the results for low laser intensity, $a_{0}=0.01$, and the lower panel depicts the nonlinear regime with $a_{0}=0.7$ for a laser pulse length $\sigma=18$ in both cases. The parameters are: black: $(\gamma, \alpha, \varphi, \theta, \phi)=(80,0,0,0,0)$; green: $(65,0.002,0$, $0.002,0.03)$; yellow: $(105,0.005,0.2,0.005,0.5)$; blue: (95, $0.001,0.3,0.003,0)$; orange: $(130,0.001,0.3,0.01,0)$; red: $(280,0.01, \pi / 2,0.01, \pi)$. The orange and red curves are only shown in the upper panel. The black dashed curves beneath each colored curve are obtained by transforming the black solid curve to the respective new parameters with (23), thus demonstrating the accuracy of the scaling law.

$$
\rho_{W}\left(\omega^{\prime} ; n^{\prime}\right)=\sum_{i=1}^{N_{e}} \rho\left(\omega^{\prime} ; n^{\prime}, u^{(i)}\right) .
$$

Each individual electron has a certain initial 4-velocity $u^{(i)}$, thus the warm spectral density contains information on the electron phase-space distribution prior to emission. For a realistic electron beam, the initial energies $\gamma^{(i)}$ and incidence angles $\left(\alpha^{(i)}, \varphi^{(i)}\right)$ of the individual electrons are distributed in some way around a mean velocity $\mathbf{u}_{0}=$ $\mathbf{u}\left(\gamma_{0}, \alpha_{0}, \varphi_{0}\right)$. To estimate the effects that a distributed electron bunch has on the spectrum in a fixed observation direction $n^{\prime}$, we just have to consider variations of the velocity $u$ around $u_{0}$. For a large number of electrons, it is more efficient to work with smooth electron phase-space distribution functions [11], so that (27) is transformed into

$$
\rho_{W}\left(\omega^{\prime} ; n^{\prime}\right)=N_{e} \int d(\alpha, \varphi, \gamma) f(\alpha, \varphi, \gamma) \rho\left(\omega^{\prime} ; n^{\prime}, u\right)
$$

with a one-particle phase-space distribution function $f$, normalized as $\int d(\alpha, \varphi, \gamma) f(\alpha, \varphi, \gamma)=1$. By means of the scaling law (23), we obtain 


$$
\begin{aligned}
\rho_{W}\left(\omega^{\prime} ; n^{\prime}\right)= & N_{e} \int d(\alpha, \varphi, \gamma) f(\alpha, \varphi, \gamma) \mathcal{M}\left(u_{0}, u\right) \\
& \times \rho\left(\omega^{\prime} / h ; n^{\prime}, u_{0}\right) .
\end{aligned}
$$

For convenience, we introduce a new rotated coordinate system attached to the mean initial velocity $\mathbf{u}_{0}$ :

$$
\begin{aligned}
& \mathbf{e}_{x^{\prime}}=-\cos \alpha_{0} \cos \varphi_{0} \mathbf{e}_{x}-\cos \alpha_{0} \sin \varphi_{0} \mathbf{e}_{y}+\sin \alpha_{0} \mathbf{e}_{z}, \\
& \mathbf{e}_{y^{\prime}}=\sin \varphi_{0} \mathbf{e}_{x}-\cos \varphi_{0} \mathbf{e}_{y}, \\
& \mathbf{e}_{z^{\prime}}=\sin \alpha_{0} \cos \varphi_{0} \mathbf{e}_{x}+\sin \alpha_{0} \sin \varphi_{0} \mathbf{e}_{y}+\cos \alpha_{0} \mathbf{e}_{z},
\end{aligned}
$$

i.e. in the new coordinate system $\mathbf{u}_{0}^{\prime}=\left(0,0, \beta_{0} \gamma_{0}\right)$ and $\alpha_{0}^{\prime}=\varphi_{0}^{\prime}=0$. The distribution functions are defined in the primed coordinate system via $f\left(\gamma, \alpha^{\prime}, \varphi^{\prime}\right)=$ $f_{1}(\gamma) f_{2}\left(\alpha^{\prime}\right) f_{3}\left(\varphi^{\prime}\right)$ (primed angles are defined in the primed coordinate system in the same way as the unprimed angles are defined in the unprimed coordinate system) with

$$
\begin{aligned}
f_{1}(\gamma) & =\frac{1}{\sqrt{2 \pi} \Delta \gamma} \exp \left\{-\frac{\left(\gamma-\gamma_{0}\right)^{2}}{2(\Delta \gamma)^{2}}\right\} \\
f_{2}\left(\alpha^{\prime}\right) & =\frac{\alpha^{\prime}}{(\Delta \alpha)^{2}} \exp \left\{-\frac{\alpha^{\prime 2}}{2(\Delta \alpha)^{2}}\right\}, \\
f_{3}\left(\varphi^{\prime}\right) & =\frac{1}{2 \pi}
\end{aligned}
$$

where $\gamma_{0} \gg 1, \Delta \gamma \ll \gamma_{0}$ is required for $f_{1}$, and $\Delta \alpha \ll$ $\pi / 2$ is required for $f_{2}$; these constraints are typically fulfilled for relativistic electron beams.

The results for the warm spectral density $\rho_{W}\left(\omega^{\prime} ; n^{\prime}\right)$ in the linear Thomson scattering regime are compared to the cold spectral density $\rho_{C}\left(\omega^{\prime} ; n^{\prime}\right)=\rho\left(\omega^{\prime} ; n^{\prime}, u_{0}\right)$, which is $N_{e}$ times the spectral density for a single electron with velocity $u_{0}$ [i.e. the corresponding phase-space distribution would be $\left.f_{C}=\delta\left(\gamma-\gamma_{0}\right) \delta\left(\alpha-\alpha_{0}\right) \delta\left(\varphi-\varphi_{0}\right)\right]$ in Fig. 2 for different incidence angles of the electron in the linear regime, i.e. $a_{0}=0.01$. The observation direction is the mean direction of the incoming electron in each case, i.e. $\mathbf{n}^{\prime} \| \mathbf{u}_{0}$. The inherent width and shape of the peaks of the cold spectral density are determined by the width and shape of the laser envelope function $g_{\sigma}$. The effects of energy and angular spread of the incoming electron bunch are a broadening of the peaks and a reduction of the peak heights. Dominantly, the peaks are shifted to the lowenergy side due to the effects of angular spread. The relative bandwidth is the same for each geometry; also the integrated value $\int \rho\left(\omega^{\prime}\right) d \omega^{\prime}$ is the same for the three spectra exhibited.

In [1] the warm spectral density for backscattering headon collisions $\left(\theta=\alpha_{0}=0\right)$ has been calculated for different sets of electron-beam parameters (cf. Fig. 10 in [1]). A set of electron-beam parameters was determined and presented in Table 1 in [1] as $m \gamma_{0}=40 \mathrm{MeV}, \Delta \gamma / \gamma_{0}=$ $10^{-3}, \Delta \alpha=1.75 \mathrm{mrad}$, which might allow for an experimental verification of the substructures due to

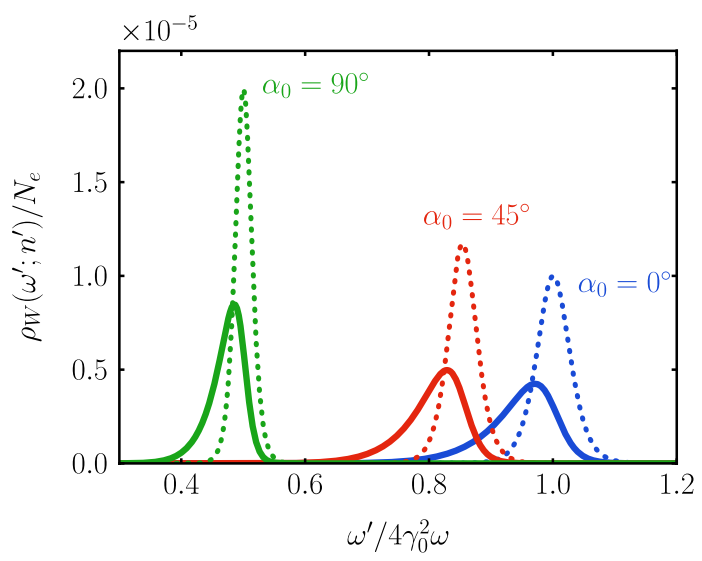

FIG. 2. Cold spectral density (dotted curves) vs warm spectral density (solid curves) at low intensity, $a_{0}=0.01$, and a laser pulse length of $\sigma=18$ observed in the direction of the incoming electron bunch $\mathbf{n}^{\prime} \| \mathbf{u}_{0}$, i.e. $\theta=\alpha_{0}, \phi=\varphi_{0}$, for different electron incidence angles $\alpha_{0}$, which are given in the plot. The spectral densities have been calculated for an energy of $m \gamma_{0}=$ $40 \mathrm{MeV}$ with an energy spread of $\Delta \gamma / \gamma_{0}=0.001$ and an angular spread of $\Delta \alpha=2.5 \mathrm{mrad}$.

ponderomotive broadening in nonlinear Thomson/ Compton scattering for this particular scattering geometry.

Here, we extend the analysis to allow for non-head-on collisions of the electron beam with the laser pulse which is much closer to an actual experimental situation. In particular, we calculate the warm spectral density-employing (29) - for the incidence angles $\alpha_{0}=10^{\circ}, 45^{\circ}$, and $90^{\circ}$. The numerical results are exhibited in Fig. 3, where one observes a decrease in the frequency of the scattered radiation (e.g., for $\alpha_{0}=90^{\circ}$ the maximum Doppler shift reaches half the value of the Doppler shift at $\alpha_{0}=0^{\circ}$ ). The contrast, however, of the minima and maxima stays

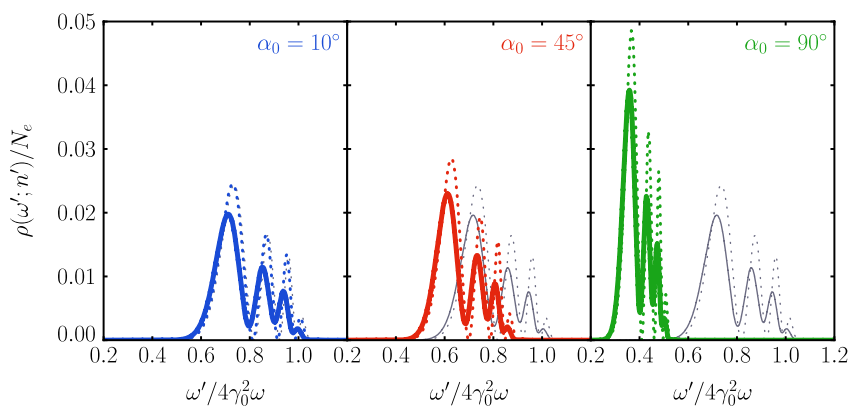

FIG. 3. Dependence of the warm spectral density (thick solid curves) on the scaled photon frequency for different electron angles of incidence $\alpha_{0}=10^{\circ}, 45^{\circ}$, and $90^{\circ}$. The spectrum is observed in the direction of the primary electron bunch $\mathbf{n}^{\prime} \| \mathbf{u}_{0}$, i.e. $\theta=\alpha_{0}$ and $\phi=\varphi_{0}$. For comparison, the respective cold spectral densities are also shown as colored dotted lines. To guide the eye, the results for $\alpha_{0}=0^{\circ}$ are also shown in each panel as thin gray solid and dotted curves. Parameters are $a_{0}=1.0, \sigma=18, \omega=1.5 \mathrm{eV}, m \gamma_{0}=40 \mathrm{MeV}, \Delta \gamma / \gamma_{0}=10^{-3}$, and $\Delta \alpha=1.75 \mathrm{mrad}$. 
the same in all cases. Such geometries, where the spectrum is observed in the forward direction of the electron, turn out to be equally well suited for an experimental observation of the substructures with the reported electron-beam parameters as the head-on backscattering geometry discussed in [1].

The maximum Doppler shift for an electron with incidence angles $(\alpha, \varphi)$ is reached at an observation direction characterized by the angles $\phi=\varphi$ and $\theta=\alpha+\frac{\sin \alpha}{\beta} \frac{a_{0}^{2}}{4 \gamma_{\kappa}}$. Thus, the observation angle of the maximum Doppler shift is not the incidence angle of the electron, but it is shifted by an intensity dependent correction $\propto a_{0}^{2}$ due to the buildup of quasimomentum in the laser pulse, and it is always larger than $\alpha$. For the parameters employed in this paper $\left(a_{0} \sim 1\right.$, $\gamma \sim 100)$, this deviation is of the order of $10^{-5}$, i.e., $0.001^{\circ}$ and will therefore be neglected in the following.

Going one step further, we now consider the dependence of the warm spectral density on the observation direction, slightly deviating from the forward direction of the primary electrons. The radiation scattered off an ultrarelativistic electron forms a cone with the central axis given by the direction of the electron velocity $\mathbf{u}$ and the polar angle (i.e. the opening angle of the cone) is given by the angle $\bar{\alpha}$ [cf. Eqs. (10) and (11)].

Expanding the Doppler shift function $\chi_{1}$ for small opening angles, one obtains

$$
\chi_{1}(\bar{\alpha})=\chi_{1}(0)\left(1+\frac{\lambda \chi_{1}(0)}{2} \bar{\alpha}^{2}+\mathcal{O}\left(\bar{\alpha}^{4}\right)\right)
$$

and $\bar{\alpha} \simeq \sqrt{(\alpha-\theta)^{2}+(\varphi-\phi)^{2} \sin ^{2} \alpha}$, where both $|\alpha-\theta|$ $\ll 1$ and $|\varphi-\phi| \ll 1$. This accounts for both the changes in the initial electron direction due to the emittance and the changes in the observation direction. The typical cone opening, where the Doppler factor changes its value significantly, is set by the scale $\bar{\alpha} \sim 1 / \gamma_{0}$, which is for our parameters $\bar{\alpha} \sim 10 \mathrm{mrad}$. For $\bar{\alpha} \ll 1 / \gamma_{0}$, the Doppler shift is almost constant. This explains the good visibility of the subpeaks in the geometries studied: Because the typical angular spread due to the beam emittance is much smaller than $1 / \gamma_{0}$, all electrons of the bunch provide approximately the same Doppler upshift for the spectrum observed in the direction of $\mathbf{u}_{0}$.

However, if the radiation is observed in a different direction, we cannot expect to have the same situation, since the Doppler upshift strongly depends on the direction of each electron. Indeed, our simulations using the scaling law show that the contrast of the subpeaks vanishes rapidly for off-axis observation directions. The numerical results are exhibited in Fig. 4 for $\alpha_{0}=10^{\circ}$ and an observation direction $\theta=\alpha_{0}+\delta \theta$, which slightly deviates from this direction by less than $1^{\circ}$. The contrast of the subpeaks vanishes completely for $\delta \theta \geq 0.4^{\circ}$.

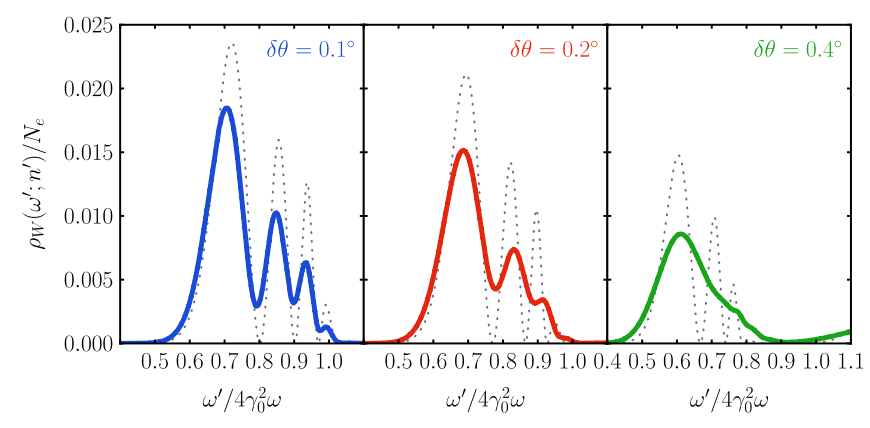

FIG. 4. Warm spectral density for an electron incidence angle $\alpha_{0}=10^{\circ}$ relative to the laser beam (as in the left panel of Fig. 3), but at a slightly different observation direction given by the angles $\delta \theta=\theta-\alpha_{0}$ and $\phi=\varphi_{0}=0$. As a comparison, the results for the respective cold spectral density are also shown as dotted curves in each panel. All other parameters are the same as in Fig. 3.

\section{FINITE DETECTOR SIZE}

Until now we always considered the detection of photons in a fixed direction $n^{\prime}$. But we have shown in the last section that the observed spectrum strongly depends on the observation direction, i.e., changes of the observation direction on the order of tenths of degrees drastically change the observed photon spectrum. Thus, it is necessary to consider the effect of a finite-size detector that collects all photons that hit a certain area since it measures the integrated spectrum over a certain range of angles $\theta$ and $\phi$ of the direction of the outgoing photon. The detector should cover only a cone around the direction of $\mathbf{u}_{0}$ with an opening angle $\delta \theta \ll 1 / \gamma_{0}$.

Employing the scaling law, the spectrum measured by a finite detector reads

$$
\begin{aligned}
\rho_{D}\left(\omega^{\prime}\right)= & N_{e} \int d \Omega d(\alpha, \varphi, \gamma) f(\alpha, \varphi, \gamma) S(\theta, \phi) \\
& \times \mathcal{M}\left(u_{0}, u, n_{0}^{\prime}, n^{\prime}\right) \rho\left(\omega^{\prime} / h, u_{0}, n_{0}^{\prime}\right),
\end{aligned}
$$

where $S(\theta, \phi)$ is the detector acceptance function, which is, in an ideal case, 1 on the surface of the detector and 0 otherwise, e.g. $S_{D}\left(\theta^{\prime}, \phi^{\prime}\right)=\Theta\left(\theta_{D}-\theta^{\prime}\right)$ with $\tan \theta_{D}=$ $D / 2 L$ for a circular detector of diameter $D$ placed at a distance $L$ away from the interaction point in the direction of $\mathbf{u}_{0}$. Examples of the photon distributions are exhibited in Fig. 5. There, the detector spectral density $\rho_{D}$ for an electron incidence angle $\alpha_{0}=10^{\circ}$ is contrasted to an approximation, where the spectrum of an idealized pointlike detector is multiplied by the respective real detector size, i.e. $\rho_{p D}=\rho_{W}\left(\omega^{\prime} ; n_{0}^{\prime}\right) \int d \Omega S(\theta, \phi)$, which is an integral mean value approximation to (33). Naturally, a larger detector collects more photons, but at the price that the contrast of the subpeaks is also reduced. For the parameters considered here, the active detector area should 

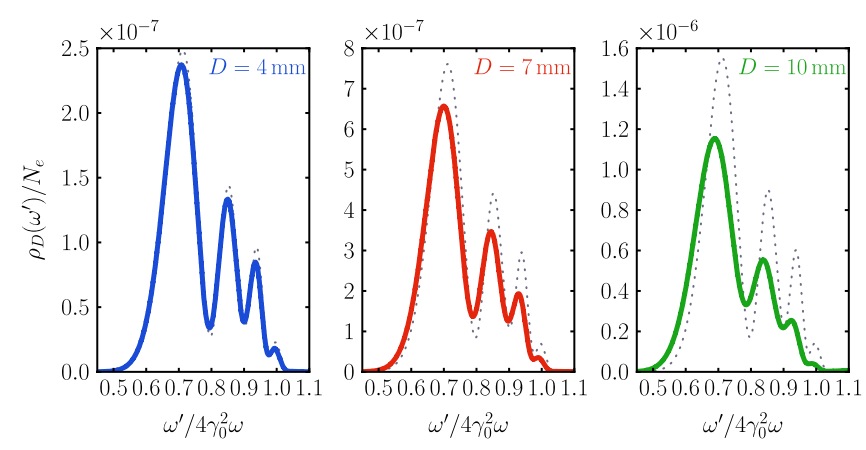

FIG. 5. Photon spectrum for a finite detector size. The solid curves correspond to a detector geometry as described below Eq. (33) with diameter $D=4 \mathrm{~mm}$ (left panel), $7 \mathrm{~mm}$ (center panel), and $10 \mathrm{~mm}$ (right panel) at a distance of $L=1 \mathrm{~m}$ from the interaction point in the mean direction of the primary electron beam for an electron incidence angle $\alpha_{0}=10^{\circ}$. For comparison, the spectra of an idealized pointlike detector $\rho_{p D}$ normalized to the same effective size are also plotted as dotted lines in each panel.

be much smaller than $10 \mathrm{~mm}$ in diameter, corresponding to a cone opening angle $\delta \theta \ll 5 \mathrm{mrad}$.

\section{SUMMARY AND CONCLUSION}

In summary, we present a general scaling law of the photon spectral density in nonlinear Thomson/Compton scattering within a plane-wave model for a laser pulse of finite duration. The scaling law provides a suitable tool for averaging over the electron-beam phase-space distribution, hence to model realistic electron beams. With the generalized version of the scaling law we are able to simulate realistic Thomson/Compton scattering experiments with arbitrary side-injection geometries for the first time within this model. Furthermore, finite detector sizes can easily be accounted for by our scaling law.

We apply the scaling law to elucidate under which circumstances the predicted substructures in the nonlinear Thomson/Compton scattering can be verified experimentally. We conclude that the photon spectrum should be observed in the direction of the primary electron beam $\mathbf{u}_{0}$ to minimize the distorting effects of the electronbeam emittance on the photon spectral density. This behavior can be understood by studying the angular dependence of the Doppler upshift factor for observation directions given by the mean electron-beam direction.

An electron beam with parameters as presented in [1] should be adequate to allow for an experimental verification of the substructures supposing the spectrum is measured in the very direction of the primary electron beam. Only few tenths of degrees away from this direction, the contrast between the minima and maxima vanishes for otherwise identical parameters.

Our scaling law allows also for an easy consideration of finite-size detectors, where one has to integrate over a certain radiation cone. A detector should cover a sufficiently narrow cone around the direction of the incoming electron beam with an opening angle much smaller than the inverse Lorentz factor. In our analysis, opening angles of 2-3 mrad (for a $40 \mathrm{MeV}$ electron beam) yield good results with suitable contrast of minima and maxima, thus offering the conditions for an experimental setup appropriate for verifying the predicted substructures in nonlinear Thomson/Compton scattering.

\section{ACKNOWLEDGMENTS}

The authors thank T.E. Cowan, T. Heinzl, and K. Chouffani for valuable discussions.

[1] T. Heinzl, D. Seipt, and B. Kämpfer, Phys. Rev. A 81, 022125 (2010).

[2] V.B. Berestetsky, E. M. Lifshitz, and L.P. Pitaevsky, Quantum Electrodynamics (Course of Theoretical Physics) (Pergamon, Oxford, UK, 1982), Vol. 4.

[3] D. Yu. Ivanov, G. L. Kotkin, and V. G. Serbo, Acta Phys. Pol. B 37, 1073 (2006).

[4] E. Esarey et al., Phys. Rev. E 48, 3003 (1993).

[5] C. Bamber et al., Phys. Rev. D 60, 092004 (1999).

[6] S.-y. Chen, A. Maksimchuk, and D. Umstadter, Nature (London) 396, 653 (1998).

[7] F. V. Hartemann et al., Phys. Rev. E 54, 2956 (1996).

[8] G. A. Krafft, Phys. Rev. Lett. 92, 204802 (2004).

[9] J.D. Jackson, Classical Electrodynamics (Wiley, New York, 1975), 2nd ed.

[10] J. W. Meyer, Phys. Rev. D 3, 621 (1971).

[11] F. V. Hartemann, High-Field Electrodynamics (CRC Press, Boca Raton, 2002). 